\title{
DESSORÇÃO E LIXIVIAÇÃO DE CHUMBO EM ESPODOSSOLO TRATADO COM AGENTES QUELANTES ${ }^{(1)}$
}

\author{
Eriberto Vagner de Souza Freitas ${ }^{(2)}$, Clístenes Williams Araújo do \\ Nascimento $^{(3)}$, Caroline Miranda Biondi ${ }^{(4)}$, João Paulo Siqueira da \\ Silva $^{(5)} \&$ Adailson Pereira de Souza ${ }^{(6)}$
}

\begin{abstract}
RESUMO
A baixa degradabilidade no solo de quelantes sintéticos usados para induzir a fitoextração pode significar elevado risco ambiental pela lixiviação de metais pesados. Agentes quelantes naturais, mais rapidamente degradados no solo, podem ser uma alternativa para os quelantes sintéticos. Este trabalho objetivou avaliar a capacidade de três agentes quelantes sintéticos e dois quelantes naturais de cadeia alifática na dessorção de $\mathbf{P b}$ de um Espodossolo contaminado pela deposição de escórias resultantes da reciclagem de baterias automotivas. A lixiviação de $\mathbf{P b}$ promovida pelos quelantes foi também estudada. $O$ trabalho compreendeu dois experimentos: (1) dessorção de $\mathrm{Pb}$ utilizando as concentrações de 0, 2, 5, 10, 20 e $30 \mathrm{mmol} \mathrm{kg} \mathrm{kg}^{-1}$ dos ácidos cítrico e oxálico e dos quelantes sintéticos EDTA, DTPA e NTA; (2) lixiviação realizada em colunas de PVC com concentrações de 0,10 e

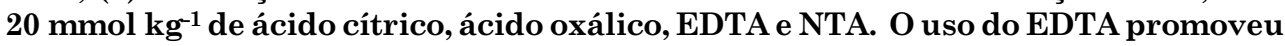
elevada dessorção e lixiviação de $\mathrm{Pb}$, não sendo recomendado para programa de fitoextração na área em estudo. A elevada dessorção de $\mathrm{Pb}$, aliada à baixa lixiviação promovida pelo NTA, qualifica o quelante para indução da fitoextração de $\mathbf{P b}$ no solo. Os quelantes naturais não apresentaram riscos para a lixiviação de $\mathrm{Pb}$ nas concentrações estudadas, mas parecem pouco promissores para a fitoextração do elemento, devido à baixa dessorção.
\end{abstract}

Termos de indexação: fitorremediação, fitoextração, contaminação do solo, ácidos orgânicos.

\footnotetext{
(1) Parte da Tese de Mestrado do primeiro autor apresentada ao Programa de Pós-Graduação em Ciência do Solo, Universidade Federal Rural de Pernambuco - UFRPE. Recebido para publicação em junho de 2008 e aprovado em março de 2009.

(2) Doutorando do Programa de Pós-Graduação em Ciência do Solo do Departamento de Agronomia. Universidade Federal Rural de Pernambuco - UFRPE. R. Dom Manoel de Medeiros s/n, Dois Irmãos, CEP 52171-900 Recife (PE). Bolsista do CNPq. Email: eribertovagner@yahoo.com.br

(3) Professor Adjunto do Departamento de Agronomia, UFRPE. E-mail: clistenes@depa.ufrpe.br

(4) Doutoranda do Programa de Pós-Graduação em Ciência do Solo do Departamento de Agronomia, UFRPE. E-mail: agrocarol@yahoo.com.br

${ }^{(5)}$ Graduando em Agronomia, UFRPE. E-mail: jprsp@yahoo.com.br

${ }^{(6)}$ Professor Adjunto do Departamento de Solos e Engenharia Rural, Universidade Federal da Paraíba - UFPB. Campus II, CEP 58397-000 Areia (PB). E-mail: adailson@cca.ufpb.br
} 


\title{
SUMMARY: LEAD DESORPTION AND LEACHING IN A SPODOSOL AMENDED WITH CHELANT AGENTS
}

\begin{abstract}
Synthetic chelants used to induce phytoextraction are barely degraded in the soil and pose high environmental risks owing to metal leaching. Natural chelating agents can be an alternative to synthetic chelates thanks to their rapid biodegrability. The study was carried out to evaluate the efficiency of $\mathrm{Pb}$ desorption of three synthetic chelators and two natural aliphatic acids from a soil contaminated with $\mathrm{Pb}$ from a car battery recycling. Additionally, effects of the amendments on Pb leaching from soil were also studied. The study was based on two experiments carried out to investigate: (1) Pb desorption from soil using 0, 2, 5, 10, 20, and $30 \mathrm{mmol} \mathrm{kg}^{-1}$ of citric and oxalic acids, and synthetic chelators EDTA, DTPA and NTA; (2) Pb leaching from soil columns by applying citric and oxalic acids, EDTA, and NTA to the soil at concentrations of 0 , 10, and $20 \mathrm{mmol} \mathrm{kg}^{-1}$. EDTA application resulted in the highest $\mathrm{Pb}$ desorption and leaching; the substance is therefore not recommended for phytoextraction programs in the area. NTA caused high $\mathrm{Pb}$ desorption along with low leaching, which qualifies the substance for enhancing $\mathrm{Pb}$ phytoextraction in field conditions. Neither citric nor oxalic acid posed risks regarding $\mathrm{Pb}$ leaching. On the other hand, they seem little promising for enhancing Pb phytoextraction in view of the low $\mathrm{Pb}$ desorption.
\end{abstract}

Index terms: phytoremediation, phytoextraction, soil contamination, organic acids.

\section{INTRODUÇÃO}

Em várias regiões do mundo, a poluição do solo com metais pesados devido à intensificação das atividades industriais, agrícolas e urbanização é um problema crescente e responsável por sérios impactos ao ambiente. A poluição do solo com esses agentes decorre, principalmente, da mineração, indústria metalúrgica, disposição de resíduos tóxicos e práticas agrícolas, como aplicação de lodos e fertilizantes inorgânicos (Camelo et al., 1997; Chen et al., 2004; Mendes et al., 2006). A acumulação desses elementos em solos pode conduzir não apenas à diminuição da produtividade das culturas em decorrência de seus efeitos fitotóxicos, mas também a efeitos deletérios à saúde humana e animal.

Solos contaminados por metais pesados exigem ação remediadora, que diminua os teores desses poluentes a teores ambientalmente seguros. A fitoextração é uma técnica que usa plantas capazes de acumular elevados teores de metais pesados nos seus tecidos. Em razão do baixo custo, a técnica tem um grande potencial para remediação in situ em grandes áreas com baixo ou médio nível de contaminação. As plantas saturadas com metal podem passar por tratamento térmico para redução de biomassa e consequente disposição final ou ser utilizadas para reciclagem do metal (Nascimento \& Xing, 2006). Para o emprego da fitoextração de $\mathrm{Pb}$, uma das principais dificuldades é a manutenção de elevados teores do metal na solução do solo, devido à baixa solubilidade natural do metal (Pereira et al., 2007). Para isso, têm sido utilizados agentes quelantes sintéticos, como EDTA (ácido etilenodiaminotetra-acético), DTPA (ácido dietilenotriaminopenta-acético) e NTA (ácido nitrilotriacético), para promover a dessorção de $\mathrm{Pb}$ ligado à matriz do solo, formando um complexo solúvel e absorvível pela planta (Shen et al., 2002).
No entanto, o mais utilizado desses quelantes (EDTA) solubiliza metais em quantidades maiores que a capacidade de absorção das plantas e tem baixa biodegradabilidade. Isso resulta na manutenção de elevados teores de metais solúveis no solo por longo período, o que aumenta os riscos de lixiviação (Meers et al., 2004). O NTA, por outro lado, apresenta características favoráveis à fitoextração, como rápida degradação, baixa fitotoxicidade e alta capacidade de complexação de metais (Ruley et al., 2006). Este quelante foi mais efetivo que EDTA e DTPA na fitoextração de As e Zn em solo (Chiu et al., 2005).

Ácidos orgânicos de baixo peso molecular, como os ácidos cítrico e oxálico, apresentam a vantagem de serem mais rapidamente degradados no solo quando comparados aos quelantes sintéticos, evitando contaminação dos lençóis freáticos. No entanto, sua eficiência na fitoextração de metais tem sido geralmente menor que a obtida pelo uso de quelantes sintéticos (Nascimento et al., 2006). Jean et al. (2007) verificaram maior eficiência do ácido cítrico do que o EDTA na absorção de Cr por plantas de Datura innoxia. Entretanto, alguns trabalhos mostraram que a aplicação de $10 \mathrm{mmol} \mathrm{\textrm {kg } ^ { - 1 }}$ de ácido cítrico não foi eficiente na remoção de $\mathrm{Pb}$ (Melo et al., 2006; Nascimento et al., 2006). Quartacci et al. (2005) observaram que o NTA foi mais eficiente que o ácido cítrico em induzir o acúmulo de $\mathrm{Cd}$ na parte aérea de Brassica juncea.

$\mathrm{O} \mathrm{Pb}$ é um elemento extremamente estável no solo e altamente tóxico para seres humanos e animais, sendo classificado como o segundo mais perigoso elemento na lista de prioridade da agência de proteção ambiental americana (ATSDR, 2008). No Brasil existem várias áreas contaminadas por esse metal. Por exemplo, em Santo Amaro da Purificação, Bahia, foi constatada contaminação humana e do solo nas antigas instalações de uma empresa de refinamento e 
beneficiamento de $\mathrm{Pb}$ (Sánchez \& Anjos, 2001). Essa mesma empresa foi responsável pela contaminação de $\mathrm{Pb}$ em crianças no Vale do Ribeira (Paoliello et al., 2002). O grande crescimento mundial da indústria automobilística, com a concomitante ampliação do mercado para as baterias, que utiliza cerca de $70 \%$ do $\mathrm{Pb}$ consumido mundialmente, tornou a reciclagem destas outra das principais formas de contaminação dos solos por $\mathrm{Pb}$ (Paoliello \& Chasin, 2001).

Diante dos resultados controversos de eficiência dos quelantes e em busca de um agente indutor da fitoextração ambientalmente adequado, este trabalho objetivou avaliar a capacidade de três quelantes sintéticos (EDTA, DTPA e NTA) e dois naturais de cadeia alifática (ácidos cítrico e oxálico) na dessorção de $\mathrm{Pb}$ no solo. Adicionalmente, o risco de lixiviação desse metal após a aplicação de quelantes em solo contaminado com $\mathrm{Pb}$ proveniente de resíduos de baterias automotivas foi também estudado.

\section{MATERIAL E MÉTODOS}

O solo utilizado nos experimentos foi proveniente de uma área de deposição de escórias de uma empresa recicladora de baterias automotivas. O solo, classificado como Espodossolo Cárbico hidromórfico, foi coletado $(0-20 \mathrm{~cm})$ e caracterizado química e fisicamente (Embrapa, 1997, 1999) (Quadro 1). O teor de $\mathrm{Pb}$ no solo foi obtido por água-régia (Abreu et al., 2001).

\section{Experimento I: Dessorção de Pb no solo}

$\mathrm{Na}$ dessorção de $\mathrm{Pb}$ foram utilizados agentes quelantes com diferentes características químicas: três quelantes sintéticos (EDTA - ácido etilenodiamino-tetra-

Quadro 1. Principais características químicas e físicas do solo utilizado nos experimentos

\begin{tabular}{|c|c|}
\hline Característica & Valor \\
\hline pH (água 1:2,5) & 3,63 \\
\hline $\mathrm{Al}^{3+}\left(\mathrm{cmol}_{\mathrm{c}} \mathrm{dm}^{-3}\right)$ & 0,80 \\
\hline $\mathrm{Ca}^{2+}\left(\mathrm{cmol}_{\mathrm{c}} \mathrm{dm}^{-3}\right)$ & 0,65 \\
\hline $\mathrm{Mg}^{2+}\left(\mathrm{cmol}_{\mathrm{c}} \mathrm{dm}^{-3}\right)$ & 0,57 \\
\hline $\mathrm{P}\left(\mathrm{mg} \mathrm{dm}^{-3}\right)$ & 3,24 \\
\hline $\mathrm{K}^{+}\left(\mathrm{cmol}_{\mathrm{c}} \mathrm{dm}^{-3}\right)$ & 0,03 \\
\hline $\mathrm{Na}\left(\mathrm{cmol}_{\mathrm{c}} \mathrm{dm}^{-3}\right)$ & 0,27 \\
\hline $\mathrm{H}+\mathrm{Al}\left(\mathrm{cmol}_{\mathrm{c}} \mathrm{dm}^{-3}\right)$ & 5,06 \\
\hline Carbono orgânico (g kg-1) & 9,21 \\
\hline $\mathrm{Pb}$ total $\left(\mathrm{mg} \mathrm{dm}^{-3}\right)$ & 544 \\
\hline Areia $\left(\mathrm{g} \mathrm{kg}^{-1}\right)$ & 948 \\
\hline Silte $(\mathrm{g} \mathrm{kg} \cdot 1)$ & 22 \\
\hline Argila (g kg-1) & 30 \\
\hline $\mathrm{Ds}\left(\mathrm{g} \mathrm{cm}^{3}\right)$ & 1,64 \\
\hline
\end{tabular}

acético, DTPA - ácido dietilenotriaminopenta-acético e NTA - ácido nitrilotriacético) e dois naturais de cadeia alifática (os ácidos cítrico e oxálico). Para avaliar o efeito dos quelantes foram utilizadas as doses de $0,2,5,10,20$ e $30 \mathrm{mmol} \mathrm{kg}{ }^{-1}$, aplicadas na forma sólida a 5 g do solo contaminado. Em seguida, foram adicionados $30 \mathrm{~mL}$ de uma solução de $\mathrm{CaCl}_{2}$ $10 \mathrm{mmol} \mathrm{L}^{-1} \mathrm{em}$ tubos de centrífuga com capacidade para $50 \mathrm{~mL}$ (Nascimento, 2006).

Para promover a dessorção do metal, as amostras foram dispostas em mesa horizontal e agitadas por um período de $24 \mathrm{~h}$ a $200 \mathrm{rpm}$. Em seguida, os tubos foram centrifugados em $1.600 \mathrm{~g}$ por $15 \mathrm{~min}$, sendo o sobrenadante rapidamente filtrado, para evitar readsorção do metal. Para determinação da concentração do $\mathrm{Pb}$, os extratos foram submetidos à leitura em espectrofotômetro de absorção atômica.

Os dados foram submetidos à análise de variância $(p<0,05)$. Equações de regressão foram ajustadas em função da concentração do quelante aplicado no solo e da concentração de $\mathrm{Pb}$ dessorvido, sendo a escolha do modelo feita com base na significância dos estimadores dos parâmetros e maiores $\mathrm{R}^{2}$. As equações foram utilizadas para calcular a concentração de cada quelante que promoveu a maior taxa de dessorção do metal. As análises estatísticas foram realizadas no software Statistical Analyses System - SAS (SAS, 1999).

\section{Experimento II: Lixiviação de $\mathrm{Pb}$ em colunas de solo}

O experimento foi realizado em colunas de PVC com dimensões de $9 \mathrm{~cm}$ de diâmetro e $20 \mathrm{~cm}$ de altura, providas de coletor de lixiviado. Lã de vidro foi posicionada sobre o solo e no fundo das colunas, para evitar fluxo preferencial e perdas de solo. Durante o preenchimento das colunas foi promovido o mesmo nível de compactação do solo, utilizando-se método semelhante ao da proveta para determinação da densidade do solo da Embrapa (1997).

O experimento seguiu o delineamento em blocos casualizados, com quatro repetições, em um arranjo fatorial $(4 \times 2)+1$, que corresponde a quatro quelantes: dois sintéticos (EDTA e NTA) e dois naturais (ácidos cítrico e oxálico), nas concentrações de 10 e $20 \mathrm{mmol} \mathrm{kg}^{-1}$, e mais um tratamento controle (solo contaminado, sem aplicação de quelante), totalizando 36 parcelas.

As amostras de solo $(1 \mathrm{~kg})$ foram previamente saturadas com água destilada para $80 \%$ da capacidade de campo. Em seguida, completou-se a saturação para $100 \%$, utilizando as soluções com os quelantes. Antes da aplicação da lâmina de água, outra camada de lã de vidro foi posicionada na superfície do solo, para evitar a formação de caminhos preferenciais da solução durante a percolação pela coluna de solo. A percolação da solução de $\mathrm{Pb}$ ocorreu durante um período de quatro a $5 \mathrm{~h}$; realizou-se a aplicação de um volume de água destilada de 1,80 L, armazenada em recipientes 
plásticos de $2 \mathrm{~L}$, com vazão aproximada de 6 -

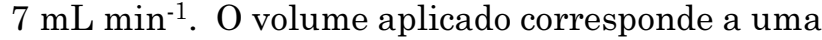
lâmina média dos meses mais chuvosos $(180 \mathrm{~mm}) \mathrm{da}$ região de coleta do solo (Rio Tinto - $\mathrm{PB}$ ), segundo dados da AESA (2006). Durante esse período, realizaram-se seis coletas de $50 \mathrm{~mL}$ e 15 coletas de $100 \mathrm{~mL}$, totalizando 21 coletas de lixiviado. $\mathrm{O}$ teor de $\mathrm{Pb}$ na solução lixiviada foi determinado por espectrofotometria de absorção atômica.

Com o objetivo de avaliar o efeito da percolação dos quelantes sobre as formas de $\mathrm{Pb}$ no solo, foi efetuada extração sequencial com base no método de Shuman (1985), com exceção da fração óxido de Fe amorfo, que foi obtida pelo método de Chao \& Zhou (1983), nas colunas em que foram aplicadas doses de $20 \mathrm{mmol} \mathrm{kg}^{-1}$ dos quelantes. Esse fracionamento separou o $\mathrm{Pb}$ nas frações trocável, matéria orgânica, óxido de Fe amorfo e óxido de Fe cristalino e foi efetuado como descrito a seguir.

Fração Trocável (Tr) - Cinco gramas de TFSA e $20,00 \mathrm{~mL}$ de $\mathrm{Mg}\left(\mathrm{NO}_{3}\right)_{2} 1 \mathrm{~mol} \mathrm{~L}^{-1}$ foram agitados por $2 \mathrm{~h}$ em um tubo de centrífuga com capacidade para $50 \mathrm{~mL}$. Em seguida, a amostra foi centrifugada, o sobrenadante filtrado e $20 \mathrm{~mL}$ de água destilada foram adicionados ao tubo. A amostra foi agitada novamente por $3 \mathrm{~min}$, centrifugada e filtrada. Os dois sobrenadantes foram combinados para análise.

Fração Matéria Orgânica (MO) - Dez mililitros de $\mathrm{NaClO} 50-60 \mathrm{~g} \mathrm{~L}^{-1}$, pH 8,5 (ajustado imediatamente antes do uso devido à sua alta instabilidade em relação ao $\mathrm{pH}$ ), foram adicionados ao tubo de centrífuga, e a amostra, aquecida em banho-maria a $100^{\circ} \mathrm{C}$ durante $30 \mathrm{~min}$, foi ocasionalmente agitada. Em seguida, a amostra foi centrifugada e o sobrenadante filtrado. Esse procedimento foi repetido duas vezes, e os três filtrados foram combinados. Após adição de $10 \mathrm{~mL}$ de água destilada, a amostra no tubo de centrífuga foi agitada por $3 \mathrm{~min}$, centrifugada e filtrada; o filtrado foi adicionado ao extrato de $\mathrm{NaOCl}$ das extrações anteriores.

Fração Óxido de Ferro Amorfo (OxFeA) - Trinta mililitros de $\mathrm{NH}_{2} \mathrm{OH} . \mathrm{HCl} 0,25 \mathrm{~mol} \mathrm{~L}^{-1}+\mathrm{HCl}$ $0,25 \mathrm{~mol} \mathrm{~L}^{-1} \mathrm{pH} 3,0$ foram adicionados à amostra no tubo de centrífuga, seguindo-se agitação por $30 \mathrm{~min}$. As amostras foram centrifugadas, filtradas e lavadas, como na extração anterior.

Fração Óxido de Ferro Cristalino $(\mathrm{OxFeC})$ - Trinta mililitros de $\left(\mathrm{NH}_{4}\right)_{2} \mathrm{C}_{2} \mathrm{O}_{4}$ (oxalato de amônio) $0,2 \mathrm{~mol} \mathrm{~L}^{-1}+\mathrm{H}_{2} \mathrm{C}_{2} \mathrm{O}_{4}$ (ácido oxálico) $0,2 \mathrm{~mol} \mathrm{~L}^{-1}+$ ácido ascórbico $0,01 \mathrm{~mol} \mathrm{~L}^{-1}, \mathrm{pH} 3,0$, foram colocados em contato com a amostra de solo no tubo de centrífuga e aquecidos por 30 minutos a $100^{\circ} \mathrm{C} \mathrm{em}$ banho-maria, sendo ocasionalmente agitados. Em seguida, as amostras foram submetidas à centrifugação e filtragem.

Os dados deste trabalho foram submetidos à ANOVA e o teste de Tukey a $5 \%$ foi utilizado para verificar a diferença entre os efeitos dos quelantes na distribuição de $\mathrm{Pb}$ nas diferentes frações do solo. Para cada dose dos quelantes, equações de regressão foram ajustadas em função do número de coletas e da concentração de $\mathrm{Pb}$ na solução lixiviada, sendo a escolha do modelo feita com base na significância dos estimadores dos parâmetros e maiores $\mathrm{R}^{2}$. As análises foram realizadas no programa Statistical Analyses System - SAS (SAS, 1999).

\section{RESULTADOS E DISCUSSÃO}

\section{Experimento I: Dessorção de $\mathrm{Pb}$ promovida por agentes quelantes}

Todos os quelantes testados tiveram efeito significativo sobre a dessorção de $\mathrm{Pb}$ do solo (Quadro 2). Entretanto, os quelantes sintéticos (EDTA, DTPA e NTA) solubilizaram concentrações de $\mathrm{Pb}$ muito maiores que os quelantes orgânicos (ácidos cítrico e oxálico) (Figura 1). A estabilidade de complexação é dependente sobretudo da afinidade química entre o metal envolvido e o quelante, afinidade essa maior para os sintéticos (Martell \& Smith, 1974). Para os quelantes orgânicos, a complexação é dependente do número e da proximidade dos grupos carboxílicos do ácido orgânico envolvido, do $\mathrm{pH}$ do solo e da concentração e do tipo de metal em questão (Pires \& Mattiazzo, 2007). Com base na taxa de recuperação de $\mathrm{Pb}$, pode-se obter a seguinte ordem na dessorção de $\mathrm{Pb}$ : EDTA > DTPA > NTA > Ácido Cítrico > Ácido Oxálico (Quadro 3).

A dessorção de $\mathrm{Pb}$ no solo alcançou taxas de recuperação que variaram de 92 a 98 \% com o uso dos quelantes sintéticos (Quadro 2). As taxas de recuperação de $\mathrm{Pb}$ com a utilização dos quelantes naturais foram bem inferiores, entre 19 e $23 \%$, respectivamente, para os ácidos oxálico e cítrico. A maior recuperação promovida pelo ácido cítrico devese à maior habilidade dos grupamentos fenólicos $(-\mathrm{OH})$ em participar das reações, visto que o ácido oxálico, mesmo apresentando maior densidade de carga, abrange apenas grupamentos carboxílicos ($\mathrm{COOH})$, menos lábeis nas reações de solubilização (Andrade et al., 2003).

A dessorção de $\mathrm{Pb}$ na solução do solo promovida pelos quelantes sintéticos foi notoriamente maior que a obtida com o uso dos quelantes naturais (Figura 1). Vários trabalhos corroboram a maior disponibilidade de $\mathrm{Pb}$ às plantas induzida por quelantes sintéticos, quando comparados aos naturais (Gupta et al., 2000; Lombi et al., 2001; Kos \& Lestan, 2004; Melo et al., 2006).

$\mathrm{O} \mathrm{Pb}$ é fortemente retido no solo, apresentando baixa mobilidade, e muito pouco é transportado para águas superficiais ou profundas (Camelo et al., 1997; Abdel-Haleem et al., 2001). Portanto, a principal restrição quanto ao uso dos quelantes sintéticos, principalmente EDTA, em condições de campo é o risco 
Quadro 2. Análise de variância das doses dos quelantes (ácido cítrico, ácido oxálico, EDTA, NTA e DTPA)

\begin{tabular}{|c|c|c|c|c|}
\hline FV & GL & SQ & QM & $\mathbf{F}$ \\
\hline & \multicolumn{4}{|c|}{ Ácido Cítrico } \\
\hline Dose & 5 & 20919,17 & 4183,83 & $42,01^{* *}$ \\
\hline Resíduo & 10 & 996,04 & 99,60 & \\
\hline \multirow[t]{2}{*}{ CV (\%) } & 10,31 & & & \\
\hline & \multicolumn{4}{|c|}{ Ácido Oxálico } \\
\hline Dose & 5 & 7955,52 & 1591,10 & $42,34^{* *}$ \\
\hline Resíduo & 10 & 375,77 & 37,57 & \\
\hline \multirow[t]{2}{*}{ CV (\%) } & 8,36 & & & \\
\hline & \multicolumn{4}{|c|}{ EDTA } \\
\hline Dose & 5 & 367829,20 & 73565,84 & $131,45^{* *}$ \\
\hline Resíduo & 10 & 5596,38 & 559,63 & \\
\hline \multirow[t]{2}{*}{ CV (\%) } & 6,85 & & & \\
\hline & \multicolumn{4}{|c|}{ NTA } \\
\hline Dose & 5 & 463569,01 & 92713,81 & $56,81^{* *}$ \\
\hline Resíduo & 10 & 16319,87 & 1631,97 & \\
\hline \multirow[t]{2}{*}{ CV (\%) } & 14,64 & & & \\
\hline & \multicolumn{4}{|c|}{ DTPA } \\
\hline Dose & 5 & 411242,41 & 82248,47 & $211,62^{* *}$ \\
\hline Resíduo & 10 & 2778,58 & 277,56 & \\
\hline CV (\%) & 5,08 & & & \\
\hline
\end{tabular}

associado à lixiviação do metal ao longo do perfil do solo (Madrid et al., 2003; Chen et al., 2004). Os valores estimados com o uso das doses de 21, 23,9 e $20,2 \mathrm{mmol} \mathrm{kg}^{-1}$ dos quelantes equivalem a concentrações de $\mathrm{Pb}$ na solução do solo de 535,0, 502,2 e $522,3 \mathrm{mg} \mathrm{L}^{-1}$, respectivamente para EDTA, NTA e DTPA (Quadro 3).

Os resultados mostram elevada dessorção de $\mathrm{Pb}$ promovida pelo NTA. Na concentração de $10 \mathrm{mmol} \mathrm{kg}{ }^{-1}$, o quelante é tão eficiente quanto EDTA e DTPA (Figura 1). Devido a características como baixa fitotoxicidade e baixa persistência no solo (Ruley et al., 2006), o NTA pode ser um quelante promissor para indução da fitoextração na área em estudo. Por exemplo, Melo et al. (2006) constataram que NTA aumentou em até 13 vezes a concentração de $\mathrm{Pb}$ na parte aérea de plantas de milho.

Os ácidos oxálico e cítrico promoveram significativa dessorção de $\mathrm{Pb}$ (Figura 1). A máxima dessorção do metal obtida com a aplicação de $30 \mathrm{mmol} \mathrm{kg}^{-1}$ dos ácidos orgânicos foi de 100,3 e $163,7 \mathrm{mg} \mathrm{L}^{-1}$, respectivamente (Quadro 3). Vários trabalhos têm ressaltado a mais baixa eficiência dos ácidos orgânicos em solubilizar metais (Renella et al., 2004; Kos \& Lestan, 2004). Segundo Nascimento
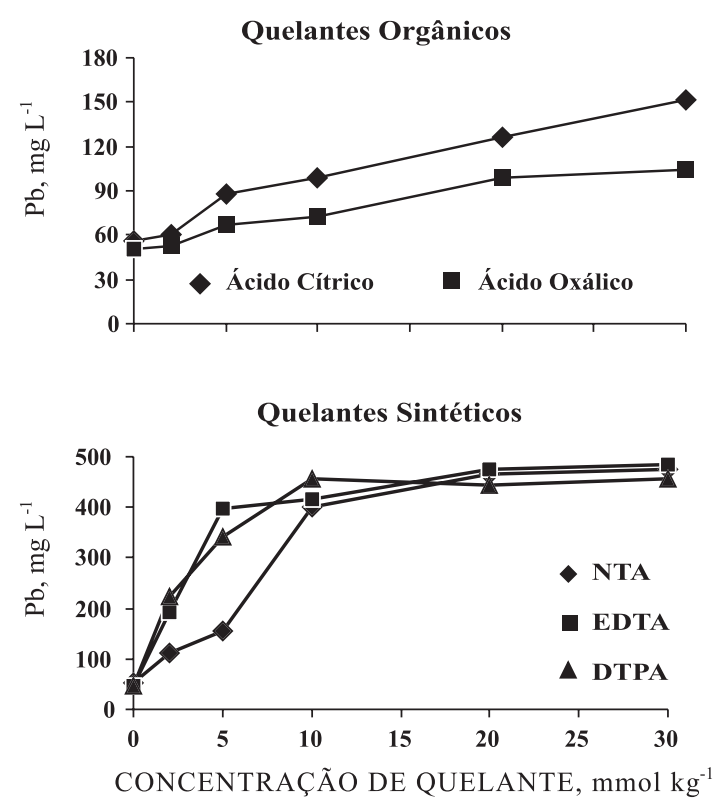

Figura 1. Efeito da aplicação dos quelantes (EDTA, NTA, DTPA, ácido cítrico e oxálico nas concentrações de $0,2,5,10,20$ e $30 \mathrm{mmol} \mathrm{kg}^{-1}$ ) na dessorção de $\mathrm{Pb}\left(\mathrm{mg} \mathrm{L}^{-1}\right)$ no solo.

Quadro 3. Equações de regressão, dose para promover a máxima dessorção, quantidade estimada de $\mathrm{Pb}$ dessorvido (em solução) e taxa de recuperação de $\mathrm{Pb}$ do quelante em relação à contaminação inicial do solo (544 $\left.\mathrm{mg} \mathrm{kg}^{-1}\right)$

\begin{tabular}{lcccc}
\hline \multicolumn{1}{c}{ Equação } & $\mathbf{R}^{2}$ & Dose & Dessorção & Recuperação \\
\hline & & $m m o l ~ k g-1$ & $\mathrm{mg} \mathrm{L}^{-1}$ & $\%$ \\
$\mathrm{~Pb}$-Ácido Ć́trico $=3,14^{* *} \mathrm{x}+61,63$ & $0,96^{* *}$ & 19,6 & 123,2 & 23 \\
$\mathrm{~Pb}$-Ácido Oxálico $=1,89^{*} \mathrm{x}+53,10$ & $0,76^{* *}$ & 28,1 & 106,2 & 19 \\
$\mathrm{~Pb}$ - EDTA $=-0,96^{* *} \mathrm{x}^{2}+40,43^{* *} \mathrm{x}+113,10$ & $0,87^{* *}$ & 21,0 & 535,0 & 98 \\
$\mathrm{~Pb}$ - NTA $=-0,81^{* *} \mathrm{x}^{2}+38,81^{* *} \mathrm{x}+37,29$ & $0,96^{* *}$ & 23,9 & 502,2 & 92 \\
$\mathrm{~Pb}$ - DTPA $=-0,99^{* *} \mathrm{x}^{2}+40,20^{* *} \mathrm{x}+115,8$ & $0,88^{* *}$ & 20,2 & 522,3 & 96 \\
\hline
\end{tabular}

** $\mathrm{e}^{*}$ : significativo a 1 e $5 \%$, respectivamente. 
(2006), o uso de uma dose de $20 \mathrm{mmol} \mathrm{kg}{ }^{-1}$ dos ácidos cítrico e oxálico não foi eficiente em dessorver $\mathrm{Pb}(5 \mathrm{e}$ $40 \mathrm{mg} \mathrm{L}^{-1}$, respectivamente). No presente trabalho, no entanto, o baixo teor de argila do solo $\left(30 \mathrm{~g} \mathrm{~kg}^{-1}\right)$ pode explicar o melhor desempenho dos ácidos, uma vez que em solos mais argilosos ocorre maior adsorção do quelante e, consequentemente, menor dessorção de $\mathrm{Pb}$.

\section{Experimento II: Lixiviação de $\mathrm{Pb}$ promovida por agentes quelantes}

Os valores totais de $\mathrm{Pb}$ lixiviado sugerem a seguinte ordem de potencial de lixiviação pelos quelantes: EDTA > NTA > ácido cítrico > ácido oxálico (Quadro 4). Em relação à contaminação inicial do solo (544 $\left.\mathrm{mg} \mathrm{kg}^{-1}\right)$, a concentração mais elevada de cada quelante resultou na lixiviação de aproximadamente 83, 42, 3 e 1,5\% do total de $\mathrm{Pb}$ do solo, respectivamente.

A aplicação dos ácidos cítrico e oxálico na concentração de $20 \mathrm{mmol} \mathrm{kg}{ }^{-1}$ promoveu aumento da lixiviação de $\mathrm{Pb}$ de três e duas vezes, respectivamente, em relação à média dos tratamentos que não receberam quelantes. Já a mesma dose de EDTA aumentou a concentração de $\mathrm{Pb}$ lixiviado em 112 vezes. Esses resultados mostram que o uso de quelantes orgânicos pode ser considerado ambientalmente seguro, quando comparados aos quelantes sintéticos. O NTA promoveu uma lixiviação de $\mathrm{Pb} 50 \%$ menor que a do EDTA (Quadro 4). Como o NTA tem uma persistência relativamente baixa no ambiente, esse problema poder ser contornado com o parcelamento das doses, evitando altos teores solúveis de metal na solução do solo.

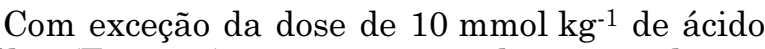
oxálico (Figura 2), que apresentou diminuição linear dos teores de $\mathrm{Pb}$ lixiviado em função das 21 coletas, os outros quelantes apresentaram comportamento quadrático na diminuição dos teores de $\mathrm{Pb}$ lixiviado. Os ácidos orgânicos demonstraram baixo risco de lixiviação de metais para o lençol freático, uma vez

Quadro 4. Somatório total de $\mathrm{Pb}$ lixiviado pela aplicação das doses de 0,10 e $20 \mathrm{mmol} \mathrm{kg}{ }^{-1}$ dos quelantes EDTA, NTA, ácido cítrico e ácido oxálico após a percolação de $1.800 \mathrm{~mL}$ de água destilada na coluna de solo contaminado

\begin{tabular}{lrrr}
\hline \multirow{2}{*}{ Quelante } & \multicolumn{3}{c}{ Concentração } \\
\cline { 2 - 4 } & $\mathbf{0}$ & $\mathbf{1 0}$ & \multicolumn{1}{c}{$\mathbf{2 0}$} \\
\cline { 2 - 4 } & $\mathrm{mg} \mathrm{L}^{-1}$ & \\
EDTA & $4 \mathrm{aC}$ & $309 \mathrm{aB}$ & $449 \mathrm{aA}$ \\
NTA & $4 \mathrm{aC}$ & $186 \mathrm{bB}$ & $224 \mathrm{bA}$ \\
Ácido cítrico & $4 \mathrm{aB}$ & $9 \mathrm{cAB}$ & $13 \mathrm{cA}$ \\
Ácido oxálico & $4 \mathrm{aA}$ & $7 \mathrm{cA}$ & $8 \mathrm{cA}$ \\
\hline
\end{tabular}

Os valores com letras iguais, na linha, não diferem estatisticamente pelo teste de Tukey com $\mathrm{p}<0,05$. que estes mostraram baixa eficiência na solubilização de $\mathrm{Pb}$. Toda a lixiviação promovida pelos ácidos orgânicos ocorre aproximadamente nos primeiros $300 \mathrm{~mL}$ de água percolada, sugerindo sua rápida degradação no solo. As taxas do metal encontradas no lixiviado para o ácido cítrico variaram em média de 0,15 a $1,46 \mathrm{mg} \mathrm{L}^{-1}$ e 0,15 a $2,10 \mathrm{mg} \mathrm{L}^{-1}$ ao longo das 21 coletas, respectivamente para as doses de $10 \mathrm{e}$ $20 \mathrm{mmol} \mathrm{kg}^{-1}$. Comportamento muito semelhante foi observado para o ácido oxálico e para os tratamentos sem aplicação do quelante (Figura 2).

Houve incremento na lixiviação de $\mathrm{Pb}$ em função do aumento das concentrações de todos os quelantes. Esse comportamento não foi observado para as coletas, visto que a concentração de $\mathrm{Pb}$ decresceu ao longo destas, exceto para o NTA. Segundo Karhu et al. (1999), o NTA apresenta menor solubilidade $(\log \mathrm{S}=$ 16,36) quando comparado a outros quelantes sintéticos, como EDTA $(\log \mathrm{S}=24,6)$ e DTPA $(\log \mathrm{S}=30,43)$. Ao contrário dos demais quelantes, o NTA mostrou elevação na taxa de lixiviação do metal da primeira até a décima e da primeira até a décima primeira coleta, respectivamente para as doses de 10 e $20 \mathrm{mmol} \mathrm{kg}^{-1}$ (Figura 3). Devido à maior solubilidade do EDTA, é possível perceber que a maior atuação deste ácido na lixiviação ocorreu nos primeiros $400 \mathrm{~mL}$ lixiviados, com redução das taxas a partir da segunda coleta.

Na menor concentração, $10 \mathrm{mmol} \mathrm{kg}^{-1}$ de EDTA, os valores médios de $\mathrm{Pb}$ lixiviado foram de 98, 65, 53, 44,24 e $12 \mathrm{mg} \mathrm{L}^{-1}$, respectivamente para a $1^{\mathrm{a}}, 2^{\mathrm{a}}, 3^{\mathrm{a}}$, $4^{\mathrm{a}}, 5^{\mathrm{a}}$ e $6^{\mathrm{a}}$ coletas (Figura 3 ), o que corresponde a $96 \%$ do total da lixiviação promovida por essa dose em

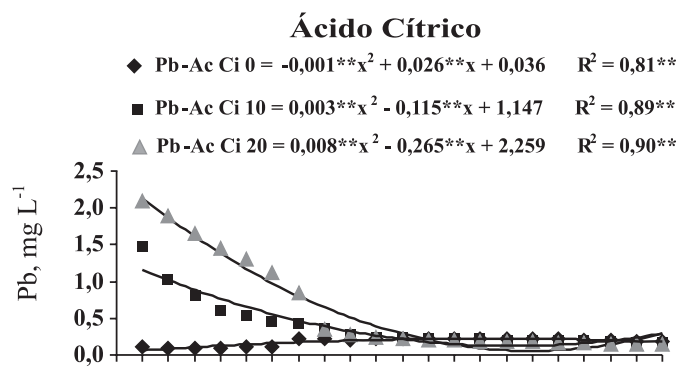

Ácido Oxálico

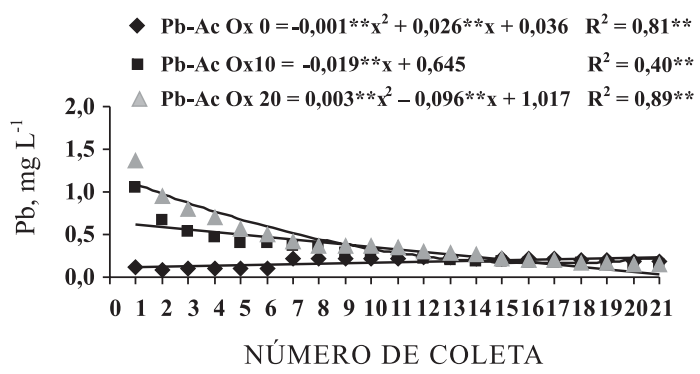

Figura 2. Concentração de $\mathrm{Pb}$ em função da aplicação das doses $\left(0,10\right.$ e $20 \mathrm{mmol} \mathrm{\textrm {kg } ^ { - 1 } )}$ dos quelantes ácidos cítrico (AC) e oxálico (AO). 


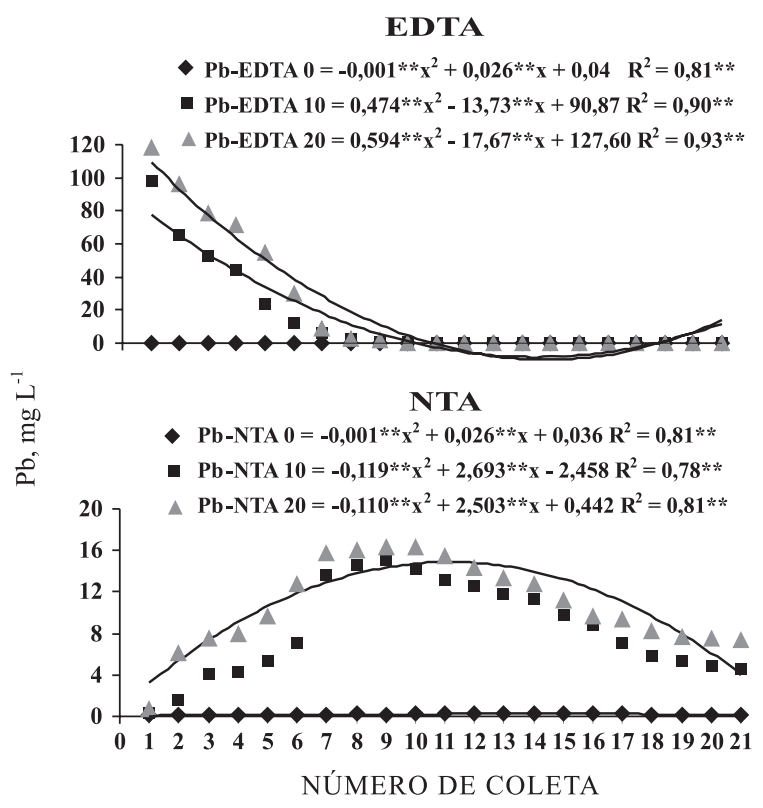

Figura 3. Concentração de $\mathrm{Pb}$ em função do número de coletas, após aplicação das doses $(0,10 \mathrm{e}$ $20 \mathrm{mmol} \mathrm{kg}^{-1}$ ) dos quelantes EDTA e NTA.

apenas $300 \mathrm{~mL}$ de água percolada pela coluna de solo. $\mathrm{Na}$ sequência, as concentrações de $\mathrm{Pb}$ alcançaram equilíbrio. Esse resultado corrobora os encontrados por Chen et al. (2004), que obtiveram as maiores concentrações de $\mathrm{Pb}$ solubilizadas pelo EDTA nos primeiros 300 e $400 \mathrm{~mL}$ lixiviados, em colunas com e sem plantas de girassol, respectivamente. Realizando o somatório das taxas deste experimento, tem-se um total de $296 \mathrm{mg} \mathrm{L}^{-1}$ de $\mathrm{Pb}$ lixiviado. Portanto, o uso de EDTA em condições de campo, em caso de evento de precipitação pluvial normalmente observada na área, demonstra a possibilidade de grande quantidade de $\mathrm{Pb}$ alcançar o lençol freático.

Os resultados demonstraram que, no ponto de máxima lixiviação com NTA, a dose de $20 \mathrm{mmol} \mathrm{kg-1}$ retirou apenas $15 \mathrm{mg} \mathrm{L}^{-1}$ de $\mathrm{Pb}$, enquanto para o EDTA, na mesma concentração, os teores de $\mathrm{Pb}$ no lixiviado foram 10 vezes maiores. Portanto, o uso de NTA, aliado a um manejo adequado (parcelamento), pode ser recomendado nas condições da área em estudo, diminuindo consideravelmente o risco de teores excessivamente elevados de $\mathrm{Pb}$ na solução.

A percolação dos quelantes provocou significativas alterações na distribuição de $\mathrm{Pb}$ entre as frações do solo. Independentemente do quelante aplicado, as concentrações de $\mathrm{Pb}$ remanescentes após a lixiviação pelos ácidos foram sempre maiores na fração trocável (Quadro 5), em razão dos baixos teores de matéria orgânica e óxidos do solo utilizado. Os teores de $\mathrm{Pb}$ em todas as frações foram maiores nos solos tratados com os quelantes orgânicos. Isto ocorreu, evidentemente, pela percolação muito maior de $\mathrm{Pb}$ além da coluna do solo, promovida pelos quelantes sintéticos. Os resultados também demonstraram que muito mais
$\mathrm{Pb}$ permaneceu no solo com o uso de NTA, em relação ao EDTA (Quadro 5). Isso pode ser explicado pela menor capacidade de solubilização do metal pelo NTA, bem como pela sua mais rápida biodegradação (Ruley et al., 2006). Esses resultados corroboram o potencial deste quelante para indução da fitoextração de $\mathrm{Pb}$ na área em estudo. Alternativamente ao EDTA, o NTA pode ser utilizado na indução da fitoextração de $\mathrm{Pb}$, com reduzido risco de lixiviação, especialmente se adotadas medidas de parcelamento na aplicação do quelante.

Quadro 5. Concentração de $\mathrm{Pb}$ remanescente nas frações do solo: trocável (Tr), matéria orgânica (MO), óxido de ferro amorfo (OxFeA) e óxido de ferro cristalino (OxFeC), após a percolação de $20 \mathrm{mmol} \mathrm{kg}^{-1}$ dos quelantes (ácidos cítrico, oxálico, EDTA e NTA)

\begin{tabular}{lrrrrrr} 
Fração & Controle & Cítrico & Oxálico & EDTA & NTA \\
\cline { 2 - 6 } & & \multicolumn{7}{c}{$\mathrm{mg} \mathrm{kg}^{-1}$} \\
\cline { 2 - 6 } $\operatorname{Tr}$ & $222,6 \mathrm{c}$ & $234,9 \mathrm{~b}$ & $265,3 \mathrm{a}$ & $26,5 \mathrm{e}$ & $170,7 \mathrm{~d}$ \\
$\mathrm{MO}$ & $42,6 \mathrm{a}$ & $17,7 \mathrm{~b}$ & $15,9 \mathrm{c}$ & $5,2 \mathrm{~d}$ & $1,7 \mathrm{e}$ \\
$\mathrm{OxFeA}$ & $44,3 \mathrm{a}$ & $30,4 \mathrm{~b}$ & $18,7 \mathrm{c}$ & $1,28 \mathrm{e}$ & $14,3 \mathrm{~d}$ \\
$\mathrm{OxFeC}$ & $44,2 \mathrm{a}$ & $18,1 \mathrm{~b}$ & $15,4 \mathrm{c}$ & $5,6 \mathrm{e}$ & $8,5 \mathrm{~d}$ \\
\hline
\end{tabular}

Os valores com letras iguais, na linha, não diferem estatisticamente pelo teste de Tukey com $\mathrm{p}<0,05$.

\section{CONCLUSÕES}

1. A alta dessorção de $\mathrm{Pb}$ com concomitante baixo potencial de lixiviação do elemento demonstra o potencial do NTA para uso em programa de fitoextração do solo. O EDTA promoveu elevada dessorção e lixiviação de $\mathrm{Pb}$, não sendo recomendado o seu uso para fitorremediação da área em estudo.

2. Os quelantes orgânicos naturais não apresentaram risco de lixiviação de $\mathrm{Pb}$, mas parecem pouco promissores para a fitoextração de $\mathrm{Pb}$ devido à baixa dessorção do metal.

\section{AGRADECIMENTOS}

O primeiro autor agradece ao CNPq e ao Programa de Pós-Graduação em Ciência do Solo da UFRPE a concessão da bolsa de estudos durante o curso de mestrado.

\section{LITERATURA CITADA}

ABDEL-HALEEM, A.S.; SCROON, A.; EL-BAHI, S.M. \& ZOHNY, E. Heavy metals and rare earth elements in phosphate fertilizer components using instrumental neutron activation analysis. App. Rad. Isot., 55:569-573, 2001. 
ABREU, M.F.; ABREU, C.A. \& ANDRADE, J.C. Determinação de fósforo, potássio, cálcio, magnésio, enxofre, cobre, ferro, manganês, zinco, níquel, cádmio, crômio e chumbo em ácido nítrico usando métodos da USEPA. In: RAIJ, B.van; ANDRADE, J.C.; CANTARELLA, H. \& QUAGGIO, J.A., eds. Análise química para avaliação da fertilidade de solos tropicais. Campinas, Instituto Agronômico de Campinas, 2001. p.251-261.

AGÊNCIA EXECUTIVA DE GESTÃO DAS ÁGUAS DO ESTADO DA PARAÍBA - AESA. Pluviometria média do Estado da Paraíba - 2006. Disponível em: <http:// www2.aesa.pb.gov.br/meteoro/pcdlmrs.shtml $>$ Acessado em 3 de nov. de 2007.

ANDRADE, F.V.; MENDONÇA, E.S.; ALVAREZ V., V.H. \& NOVAIS, R.F. Adição de ácidos orgânicos e húmicos em Latossolos e adsorção de fosfato. R. Bras. Ci. Solo, 27:10031011,2003

AGENCY FOR TOXIC SUBSTANCES \& DISEASE REGISTRY - ATSDR. Disponível em: <http://www.atsdr.cdc.gov/ cercla/05list.html> Acessado em 16 de nov. de 2008.

CAMELO, L.G.L.; MIGUEZ, S.R. \& MARBÁN, L. Heavy metals input with phosphate fertilizers used in Argentina. Sci. Total Environ., 204:245-250, 1997.

CHAO, T.T. \& ZHOU, L. Extraction techniques for selective dissolution of amorphous iron oxides from soils and sediments. Soil Sci. Soc. Am. J., 47:224-232, 1983.

CHEN, Y.; XIANGDONG, L. \& SHEN, Z. Leaching and uptake of heavy metals by ten different species of plants during an EDTA-assisted phytoextraction process. Chemosphere, 57:187-196, 2004.

CHIU, K.K.; YE, Z.H. \& WONG, M.H. Enhanced uptake of As, $\mathrm{Zn}$, and $\mathrm{Cu}$ by Vetiveria zizanoides and Zea mays using chelating agents. Chemosphere, 60:1365-1375, 2005.

EMPRESA BRASILEIRA DE PESQUISA AGROPECUÁRIA EMBRAPA. Manual de análises químicas de solos, plantas e fertilizantes. Brasília, Embrapa Comunicação para Transferência de Tecnologia, 1999. 370p.

EMPRESA BRASILEIRA DE PESQUISA AGROPECUÁRIA EMBRAPA. Manual de métodos de análises de solo. Brasília, Embrapa Comunicação para Transferência de Tecnologia, 1997. 212p.

GUPTA, S.K.; HERREN, T.; WENGER, K.; KREBS, R. \& HARI, T. In situ gentle remediation measures for heavy metalpolluted soils. In: TERRY, N. \& BAÑUELOS, G., eds. Phytoremediation of contaminated soil and water. Boca Raton, Lewis Publisher, 2000. p.303-322.

JEAN, L.; BORDAS, F.; GAUTIER-MOUSSARD, C.; VERNAY, P.; HITMI, A. \& BOLLINGER, J.C. Effect of citric acid and EDTA on chromium and nickel uptake and translocation by Datura innoxia. Environ. Poll., 20:1-9, 2007.

KARHU, J.; HARJU, L. \& IVASKA, A. Determination of the solubility products of nitrilotriacetic acid, ethylenediaminetetraacetic acid and diethylenetriaminepentaacetic acid. Anal. Chim. Acta., 380:105-111, 1999.
KOS, B. \& LESTAN, D. Chelator induced phytoextraction and in situ soil washing of $\mathrm{Cu}$. Environ. Poll., 132:333-339, 2004.

LOMBI, E.; ZHAO, F.J.; DUNHAM, S.J. \& McGRATH, S.P. Phytoremediation of heavy metal-contaminated soils: Natural hyperaccumulation versus chemically enhanced phytoextraction. J. Environ Qual., 30:1919-1926, 2001.

MADRID, F.; LIPHADZI, M.S. \& KIRKHAM, M.B. Heavy metal displacement in chelate-irrigated soil during phytoremediation. J. Hydrol., 271:107-119, 2003.

MARTELL, W.E. \& SMITH, W.M. Critical stability constants. Amino acids. New York, Plenum Press, 1974. 469p.

MEERS, E.; HOPGOOD, M.; LESAGE, E.; VERVAEKE, P.; TACK, F.M.G. \& VERLOO, M.G. Enhanced phytoextraction: In search of EDTA alternatives. Inter. J. Phytorem., 6:95109, 2004.

MELO, E.E.C.; NASCIMENTO, C.W.A. \& SANTOS, A.C.Q. Solubilidade, fracionamento e fitoextração de metais pesados após aplicação de agentes quelantes. R. Bras. Ci. Solo, 30:1051-1060, 2006.

MENDES, A.M.S.; DUDA, G.P.; NASCIMENTO, C.W.A. \& SILVA, M.O. Bioavailability of cadmium and lead in a soil amended with phosphorus fertilizers. Sci. Agric., 63:328332,2006

NASCIMENTO, C.W.A.; AMARASIRIWARDENA, D. \& XING, B. Comparison of natural organic acids and synthetic chelates at enhancing phytoextraction of metals from a multi-metal contaminated soil. Environ. Poll., 140:114123,2006

NASCIMENTO, C.W.A. Organic acids effects on desorption of heavy metals from a contaminated soil. Sci. Agric., 63:276280,2006 .

NASCIMENTO, C.W.A. \& XING, B. Phytoextraction: A review on enhanced metal availability and plant accumulation. Sci. Agric., 63:299-311, 2006.

PAOLIELLO, M.M.B. \& CHASIN, A.A.M. Ecotoxicologia do chumbo e seus compostos. Salvador, CRA, 2001. 144p. (Cadernos de Referência Ambiental, 3)

PAOLIELLO, M.M.B.; CAPITANI, E.M.; CUNHA, F.G.; MATSUO, T.; CARVALHO, M.F.; SAKUMA, A. \& FIQUEIREDO, B.R. Exposure of children to lead and cadmium from a mining area of Brazil. Environ. Res., 88:120-128, 2002.

PIRES, A.M.M. \& MATTIAZZO, M.E. Cinética de solubilização de metais pesados por ácidos orgânicos em solos tratados com lodo de esgoto. R. Bras. Ci. Solo, 31:143-151, 2007.

PEREIRA, B.F.F.; ABREU, C.A.; ROMEIRO, S.; LAGÔA, A.M.M.A. \& GONZÁLEZ, A.P. Pb-phytoextraction by maize in a Pb-EDTA treated Oxisol. Sci. Agric., 64:52-60, 2007.

QUARTACCI, M.F.; BAKER, A.J.M. \& NAVARI-IZZO, F. Nitrilotriacetate and citric acid assisted phytoextraction of cadmium by Indian mustard (Brassica juncea (L.) Czernj, Brassicaceae). Chemosphere, 59:1249-1255, 2005.

RENELLA, G.; LANDI, L.L. \& NANNIPIERI, P. Degradation of low molecular weight organic acids complexed with heavy metals in soil. Geoderma, 122:311-315, 2004. 
RULEY, A.T.; SHARMA, N.C.; SAHI, S.V.; SINGH, S.R. \& SAJWAN, K.S. Effects of lead and chelators on growth, photosynthetic activity and $\mathrm{Pb}$ uptake in Sesbania drummondii grown in soil. Environ. Poll., 144:11-18, 2006.

SÁNCHEZ, L.E. \& ANJOS, J.Â.S.A. Plano de gestão ambiental para sítios contaminados por resíduos industriais - $\mathrm{O}$ caso da Plumbum em Santo Amaro da Purificação/BA. Bahia Análise Dados, 10:306-309, 2001.
SAS Institute. Statistical analysis system. Procedure guide for personal computer. Cary, 1999.

SHEN, Z.G.; LI, X.D.; WANG, C.C.; CHEN, H.M. \& CHUA, H. Lead phytoextraction from contaminated soil with highbiomass plant species. J. Environ. Qual., 31:1893-1900, 2002.

SHUMAN, L.M. Fractionation method for soil microelements. Soil Sci., 140:11-22, 1985. 
